\title{
Study protocol of a randomized controlled trial of home-based computerized executive function training for children with cerebral palsy
}

\author{
María García-Galant 1,2,3, Montse Blasco 1,2,3, Lee Reid ${ }^{4}$, Kerstin Pannek ${ }^{4,5}$, David Leiva ${ }^{6}$, Olga Laporta-Hoyos ${ }^{1,2,3}$, \\ Júlia Ballester-Plané ${ }^{1,2,3}$, Júlia Miralbell ${ }^{1,2,3}$, Xavi Caldú ${ }^{1,2,3}$, Xènia Alonso ${ }^{7}$, Esther Toro-Tamargo ${ }^{8}$, \\ Mar Meléndez-Plumed ${ }^{8}$, Francisca Gimeno ${ }^{9}$, Marc Coronas ${ }^{10,11}$, Emili Soro-Camats ${ }^{10,11}$, Roslyn Boyd ${ }^{5}$ and \\ Roser Pueyo ${ }^{1,2,3^{*}}$
}

\begin{abstract}
Background: Cerebral palsy (CP) is frequently associated with specific cognitive impairments, such as executive dysfunction which are related to participation and quality of life (QOL). The proposed study will examine whether a computerized executive function (EF) training programme could provide superior benefits for executive functioning, participation, QOL and brain plasticity, as compared to usual care.
\end{abstract}

Methods: A single-blind randomized controlled trial (RCT) design will be performed. Thirty children with $\mathrm{CP}$ aged 8 to 12 years will participate in a home-based computerized multi-modal executive training programme (12 weeks, 5 days a week, $30 \mathrm{~min}$ a day training, total dose $=30 \mathrm{~h}$ ). Thirty children with $\mathrm{CP}$ matched by age, sex, motor and intelligence quotient (IQ) will compose the waitlist group. Cognitive, behavioural, emotional, participation and QOL measures will be obtained at three time points: before, immediately after and 9 months after completing the training. Additionally, structural and functional (resting state) magnetic resonance images (MRI) will be obtained in a subsample of 15 children from each group. Outcomes between groups will be compared following standard principles for RCTs.

Discussion: The study will test whether the cognitive training programme exerts a positive effect not only on neuropsychological and daily functioning of children with CP but also on other measures such as participation and QOL. We will also use brain MRI to test brain functional and structural changes after the intervention. If this on-line and home-based training programme proves effective, it could be a cost-effective intervention with short- and long-term effects on EF, participation or QOL in CP.

Trial registration: ClinicalTrials.gov: NCT04025749. Registered 19 July 2019. Retrospectively registered.

Keywords: Cerebral palsy, Executive functions, Cognitive training, Computerized therapy, Quality of life, Neuroimaging, Participation

\footnotetext{
* Correspondence: rpueyo@ub.edu

${ }^{1}$ Departament de Psicologia Clínica i Psicobiologia, Universitat de Barcelona,

Passeig Vall d'Hebron 171, Barcelona 08035, Spain

${ }^{2}$ Institut de Neurociències, Universitat de Barcelona, Passeig Vall d'Hebron

171, Barcelona 08035, Spain

Full list of author information is available at the end of the article
}

(c) The Author(s). 2020 Open Access This article is distributed under the terms of the Creative Commons Attribution 4.0 International License (http://creativecommons.org/licenses/by/4.0/), which permits unrestricted use, distribution, and reproduction in any medium, provided you give appropriate credit to the original author(s) and the source, provide a link to the Creative Commons license, and indicate if changes were made. The Creative Commons Public Domain Dedication waiver (http://creativecommons.org/publicdomain/zero/1.0/) applies to the data made available in this article, unless otherwise stated. 


\section{Background}

Cerebral palsy (CP) describes a group of permanent disorders of the development of movement and posture, causing activity limitations that are attributed to nonprogressive disturbances that occurred in the developing fetal or infant brain [1]. At present, CP is the leading cause of physical disability in children, with a prevalence of two or three out of every thousand newborns [2]. As a chronic condition, individuals with $\mathrm{CP}$ usually require lifetime medical, psychological, educational and social support [3]. People with CP present with alterations in sensation, perception, cognition, communication and behaviour that hinder activities of daily life, participation and quality of life (QOL) $[1,4,5]$.

Cognitive functioning is the result of a complex interplay between neurological, motor and communication processes as well as environmental support over time [6]. The neuropsychological profile in CP is heterogeneous with a high prevalence of visuospatial [7] and executive function (EF) deficits [6]. Regarding EF, some studies have highlighted lower performance in sustained attention, working memory, inhibition, processing speed, metacognition and strategic planning $[5,6,8,9]$.

Executive functions play an important role in behaviour regulation, social abilities and performance of activities of daily living. In this way, poor executive functioning may lead to slower social development and behavioural problems. Executive functioning is also essential for social problem solving and emotional regulation in children and young people with $\mathrm{CP}[3,9]$. EF has been shown to play an important role in the QOL of people with CP [10].

\section{Home-based multi-modal computerized training in CP}

Research in CP to date has mainly focused on medical- and movement-related interventions [11]. Cognitive interventions are however also needed given that cognitive impairment such as executive dysfunctions is usually associated with CP and may be related to participation and QOL.

Most current interventions require the participant to travel to receive their training, which may reduce adherence and efficacy, especially in people with motor disorders. Alternative EF home-based therapies, such as internetdelivered programmes or active videogames, are emerging as a popular modality to increase participants' motivation [12]. Some authors suggest that home-based computerized cognitive training has the potential to deliver novel, engaging and intensive therapies to children that could improve EF performance and participation in more complex activities and their QOL $[3,4]$. Computerized EF training has been demonstrated to improve EF domains such as inhibition, working memory and visuoperceptual processing in children born preterm, or with medical conditions such as Attention deficit hyperactivity disorder (ADHD), arterial ischaemic stroke, cranioencephalic trauma or cancer [13-17].
Similar results have been found in adults after computerized therapies [18-22].

According to Diamond there are three core EFs (inhibition, working memory and cognitive flexibility) and from these, higher-order EFs are built (reasoning, problem solving and planning \& monitoring) [23]. There have been some home-based randomized controlled trials (RCT) in children with CP focused on a single domain of EF such as working memory using the Cogmed System [24] and attention using the MiYoga Programme [25]. Previous studies in other populations indicate that training more than one EF domain allows the transference of improvements to other cognitive functions or clinical symptoms [26, 27]. For this reason, multi-modal tasks might better allow the transference of abilities across EF domains; EF interventions should consider all components and their developmental trajectories [27].

The transference effect from motor to cognitive functions has previously been tested in the CP population. Piovesana [28] used a home-based multi-modal computerized training programme designed to improve motor skills in children with CP. They were however unable to demonstrate improvements in EF performance. Some studies that used interactive training to improve motor skills in participants with CP found changes in visuoperceptual functions $[29,30]$. There is a lack of studies with long-term follow-up in this field: to our knowledge, only one study [29] has measured long-term changes in cognition (visuoperceptual function) in children with $\mathrm{CP}$.

\section{Magnetic resonance imaging (MRI) related to cognitive function training}

Measuring the changes in neuroplasticity promoted by therapy would involve understanding how and for whom rehabilitation could be effective [31]. Connectivity techniques involving diffusion MRI (dMRI) and the resting state in functional MRI (fMRI) [31, 32] have been used after motor-focused interventions in people with unilateral CP to analyse and quantify improvements. New techniques in neuroimaging might allow an understanding of the neural reorganization that occurs after a neuropsychological intervention [33].

Horowitz-Kraus [34] found that children with lector deficit increased brain activation and compensatory brain reorganization between visual and EF networks after improvements in reading skills promoted by/following computerized training. Changes in cerebral connectivity in the frontoparietal and occipital cortex networks, as well as neural connectivity changes, have also been found after cognitive training in healthy children [35-37]. Finally, Conklin [13] found changes in cerebral functioning with a reduction in the activation of the prefrontal medium and lateral cortex in children with cancer after training. In adults, some studies have found brain structural and 
functional changes in healthy participants after an intervention. Specifically, Eggenberger [22] induced modulations in prefrontal cortex oxygenation that were associated with improvements in EF. Another study found that exercise dose was associated with increased brain-derived neurotrophic factor as well as increased grey matter volume in the prefrontal and anterior cingulate cortices [38]. This study also found that memory improvements were associated with an increase in the volume of the dorsolateral prefrontal cortex.

\section{Methods}

This aim of the proposed study is to conduct a single-blind RCT with 60 children with $\mathrm{CP}$ to assess whether a homebased computerized multi-modal training programme might be effective at improving infants' EF, as the primary outcome. As secondary outcomes, the study will test whether the computerized therapy exerts a positive effect on other cognitive functions and other areas such as participation and QOL in children with CP. It is further expected that structural and functional brain changes might be observed.

The primary hypothesis to be tested is:

- The computerized multi-modal cognitive training programme will be more effective than usual care alone at improving executive functioning in children with $\mathrm{CP}$.

The secondary hypotheses are that the computerized multi-modal cognitive training programme will be more effective than usual care at improving the following outcomes in children with $\mathrm{CP}$.

The computerized multi-modal cognitive training programme will be more effective than usual care at improving the following outcomes in children with $\mathrm{CP}$ :

- Visuoperception and memory due to the transference effect.

- Participation.

- QOL.

- Structural and functional brain changes due to brain plasticity.

The long-term efficacy of the intervention will be checked. Finally, the effect of other variables such as the main sociodemographic and clinical factors on the efficacy of EF training will be evaluated.

\section{Participants}

The sample will consist of 60 children with CP. These participants will mainly be recruited from the Hospital Vall d'Hebron, the Hospital Sant Joan de Déu and the Cerebral Palsy Association ASPACE in Barcelona.

\section{Inclusion criteria}

- Aged 8-12 years.

- Manual Abilities Classification System (MACS) ranging from I to III [39].

- Intelligible yes/no response system.

- Ability to understand simple instructions as evaluated using the Screening Test of Spanish Grammar [40].

- Availability to participate in the investigation for 1 year.

- Accessibility to the internet at home.

Participants who have no metallic prostheses, brain shunts, claustrophobia or other factors that prevent the application of neuroimaging will be prioritized to undergo scanning.

\section{Exclusion criteria}

- Identified hearing or visual impairment that precludes neuropsychological assessment and cognitive training.

\section{Procedure}

Participants will be contacted by the medical staff of their reference health centre. Then, researchers will provide participant information and seek informed consent at a first interview at their reference health centre or in the study setting at the Faculty of Psychology, University of Barcelona (UB).

Assessments will be carried out at the University of Barcelona and neuroimaging will be performed at the Hospital Sant Joan de Déu. The intervention will take place in each participant's home, with monitoring and support from an experienced neuropsychologist.

\section{Design}

This study is researcher-blinded and will be a pairmatched and randomized waitlist controlled trial. Demographic data and motor, communication and other associated deficit measures will be collected at the first interview after participants' parents or guardians and children have provided informed consent. Detailed information about the methodology and timing of assessments is shown in Fig. 1. The waitlist design will ensure that all participants have access to this novel intervention by assigning each participant to either Group 1 (immediate intervention) or Group 2 (waitlist / delayed intervention).

After baseline assessments (T0, Additional file 1), the Group 2 will continue care as usual for 12 weeks, whilst the Group 1 will receive usual care as well as homebased multi- modal cognitive training for 12 weeks (30 $\mathrm{min} /$ day, 5 days/week, total of $30 \mathrm{~h}$ ). Both groups will be 


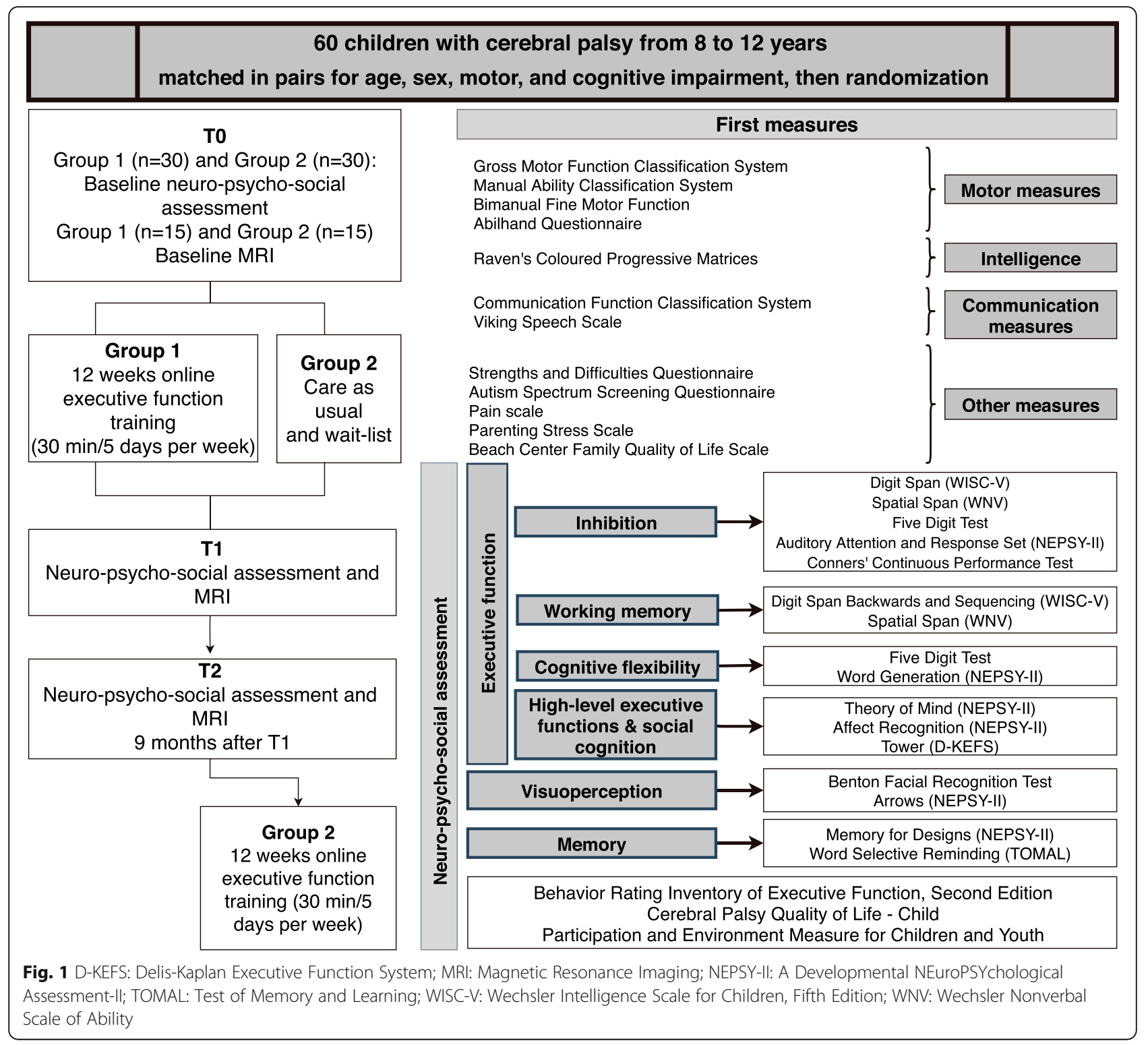

assessed after these 12 weeks (T1). A follow-up assessment will be performed 9 months after T1 (T2). After this assessment, Group 2 will have access to the same conditions as Group 1.

Patients or the public were not involved in the design, or conduct, or reporting, or dissemination of our research.

\section{Sample size determination}

The required sample size was calculated by taking into account the differences in the continuous primary outcomes after 12 weeks of following the computerized training programme. Simulations under two different scenarios were carried out. Specifically, calculations for separate tests and multiple-end-points were performed. By comparing the two scenarios, conservative figures regarding sample size were estimated. As a result, to detect a large standardized difference (i.e. a difference of at least $0.8 \mathrm{SD}$ ) between the immediate intervention and waitlist groups, with $80 \%$ power and $\alpha=0.05,26$ children would be necessary in each group. Assuming $15 \%$ attrition, the required sample size was calculated as 60 participants.

\section{Randomization}

Participants will be matched in pairs according to age, sex, IQ and manual ability. Each member of the pair will then be randomly allocated to one of the two groups. Randomization will be carried out using an in-house program written in R, DL will generate the allocation sequence and assign participants to interventions. Once the randomization process is completed, MG-G will 
inform the participants' parents and guardians of their group allocation as well as provide the details of the computerized cognitive training programme, if applicable, to their families.

\section{Blinding}

Single blinding will be applied to the researcher that performs the cognitive assessment as well as those in charge of the statistical analyses. Thus, code identification will be used for the two groups, ensuring that correspondence between codes and group characteristics (i.e. immediate intervention or waitlist) is not known by the aforementioned individuals.

\section{Adverse events}

Any minor or major events associated with the intervention or usual care groups will be screened every week through open-ended questions. Any adverse events or unintended effects detected will be reviewed by a researcher.

\section{Equipment}

The cognitive training programme requires no specialized resources other than an internet connection. All participants will receive a password to access the homebased computerized multi-modal training programme.

\section{Computerized multi-modal cognitive training}

Neuron Up (www.neuronup.com) is the cognitive training and stimulation programme that will be used. It covers all levels of difficulty of all EF domains. Table 1 shows some examples of the tasks. The training programme will also include some cognitive tasks based on activities of daily living that may promote generalization. The programme is delivered via the internet, providing the possibility of monitoring the participants' performance in real time.

A participants' instruction manual explaining how the training programme works, and providing contact information and motivational strategies to encourage engagement in the cognitive training programme, will be delivered to each participant. A trainer will be able to monitor each participant's training, and record important data such as how frequently the participant has logged into NeuronUp, how long they have spent engaged in the therapy, their training progress and their level of motivation. Neuropsychologist will contact each participant's family weekly in order to give feedback on the recorded information.

\section{Data management}

All collected data will be managed through a confidential online database run through the Universitat de Barcelona. Files containing information from the participants will be stored in a locked filing cabinet at the Universitat de Barcelona. Each participant will use a personal ID to log into NeuronUp, and the study researcher who will contact the families and follow up the training (neuropsychologist) will be different from the researcher that performs the cognitive assessment.

\section{Assessments}

Figure 1 shows the neuro-psycho-social assessments that will be performed at T0, T1 and T2. Effort has been made to select cognitive measures that are free of motor and speed components. Several domains of EF, as well as visuoperception, memory, participation and QOL will be assessed. The measures used are standardized and validated for use across a wide age range and have good retest reliability (Additional file 1).

As Sabbadini [41] advised, participants will be encouraged to answer by themselves, but some accommodations will be used if necessary, taking into account the experience of previous studies [42].

\section{MRI acquisition}

Neuroimaging will be performed at T0, T1 and T2. Participants will be prepared for the imaging sessions in order to optimize the quality of images acquired. This preparatory session will consist of explaining the procedure, familiarization with the facilities and finding entertainment strategies adapted to each participant.

Structural, functional, and diffusion MR images will be acquired on a Phillips Ingenia $3.0 \mathrm{~T}$ scanner. These sequences have been harmonised with several other studies [43-46] in order to maximise their utility and interpretability [47].

Structural imaging includes high resolution T1w MPRAGE (FOV $256 \mathrm{~mm}$; acquisition matrix size $256 \times 255$; reconstruction matrix size $256 \times 256$; 192 slices; slice thickness $1 \mathrm{~mm}$; TR $2500 \mathrm{~ms}$; TE $3.0 \mathrm{~ms}$; flip angle $9^{\circ}$; SENSE acceleration factor: RL 2) and high resolution T2w FLAIR (FOV $256 \mathrm{~mm}$; acquisition / reconstruction matrices sized $256 \times 256$; 192 slices; slice thickness $1 \mathrm{~mm}$; TR $5000 \mathrm{~ms}$; TE 388 ms; SENSE acceleration factors: AP 1.4, RL 2).

The diffusion weighted imaging protocol is a multishell sequence (FOV $240 \mathrm{~mm}$; acquisition matrix size $96 \times 94$; reconstruction matrix size $96 \times 96$; 60 slices; slice thickness $2.5 \mathrm{~mm}$; TR $8778 \mathrm{~ms}$; TE $115 \mathrm{~ms}$; flip angle $90^{\circ}$; SENSE acceleration factor: AP 2). The acquisition includes $8 \mathrm{x}$ non-diffusion weighted images $\left(\mathrm{b}=0 \mathrm{~s} / \mathrm{mm}^{2}\right)$ as well as $20\left(\mathrm{~b}=1000 \mathrm{~s} / \mathrm{mm}^{2}\right)$ and $60\left(\mathrm{~b}=3000 \mathrm{~s} / \mathrm{mm}^{2}\right)$ unique directions. These are split across four blocks with alternating $\mathrm{AP} / \mathrm{PA}$ phase encoding directions for the purpose of post-acquisition distortion correction. Acquiring images across four blocks also allows technicians to re-acquire portions of sequence if the child moves during acquisition, rather than requiring re-acquisition of the full sequence.

A resting state fMRI will also be acquired (FOV 240 $\mathrm{mm}$; acquisition matrix size $80 \times 78$; reconstruction matrix 
Table 1 Examples of multi-modal activities in home-based computerized cognitive training programme

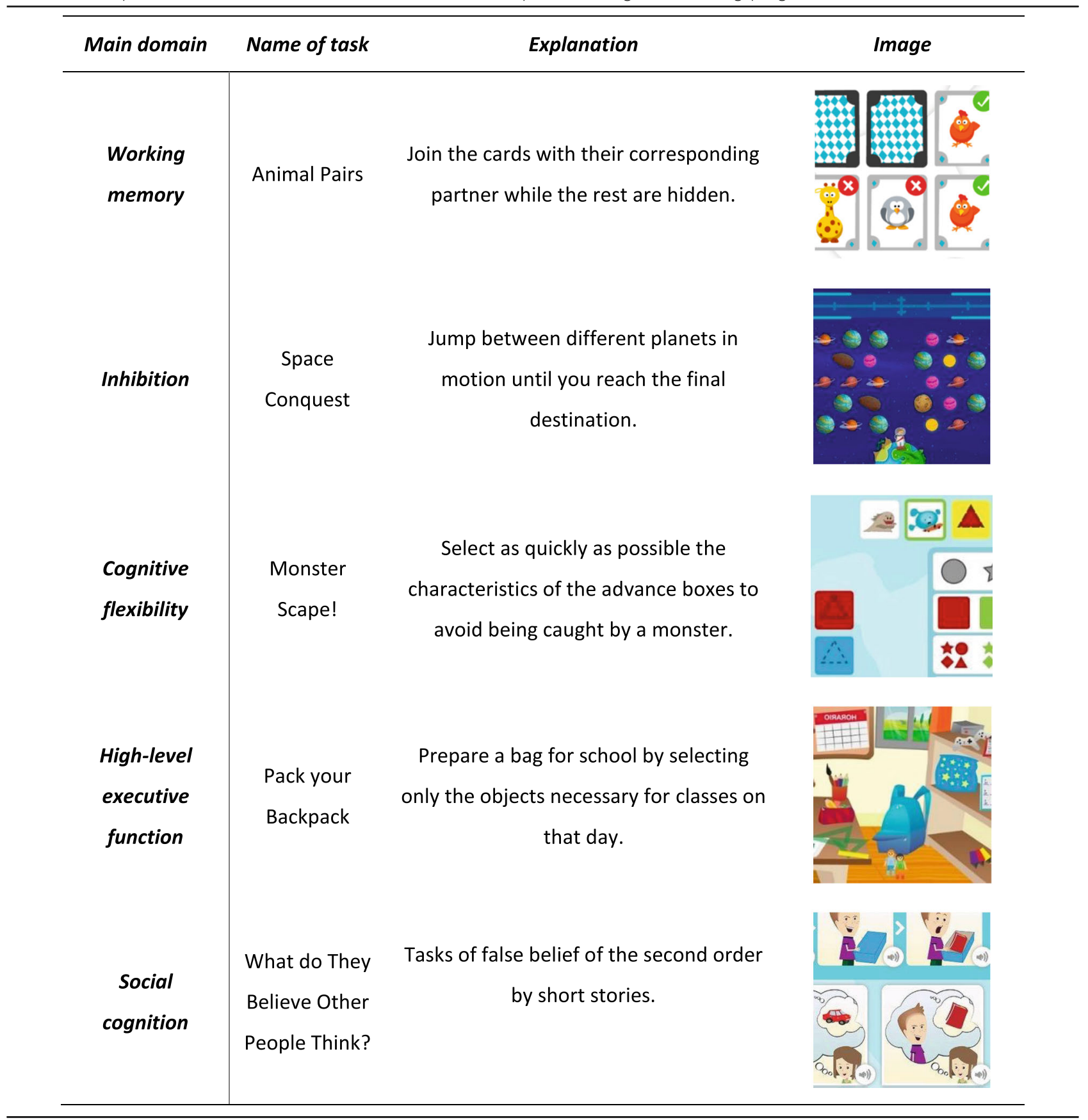

size $80 \times 80$; 40 slices; slice thickness $3 \mathrm{~mm}$; TR $2300 \mathrm{~ms}$; TE 30 ms; SENSE acceleration factor, AP 2; 266 frames). Participants will be asked to remain still and relaxed with their eyes closed for the total duration of this scan.

\section{Statistical analysis}

Statistical analysis will follow standard principles for RCTs (e.g. running sensitivity analysis with non-ignorable missing data). Primary and secondary outcomes will be summarized for each group depending on the measurement scale and data distribution (frequencies, means, SDs, medians, IQRs, 95\% CIs). To assess training effectiveness, parametric (or nonparametric, depending on the data distribution) tests will be used for the groupwise comparisons after the 12th week (post-intervention; T1). Comparisons between groups at follow-up (after 9 months; T2) will be made using Generalized Estimating Equations (GEEs). The fixed factors are time (week 0, 12, and 36), group (immediate and waitlist), and time by group interaction. Assuming a Gaussian distribution for the outcomes, identity link functions will be 
used. Given the small sample size and in order to avoid possible numerical problems, we will reduce the complexity of the model by using an exchangeable working correlation matrix. Statistical significance will be considered at $p<.05$ and post-hoc adjustment will be applied for multiple comparisons. All data will be treated following an intention-totreat approach and multiple imputation will be used for treating missing data whenever possible.

Finally we will compare neuroimaging data achieved from structural sequences as T1w, T2w FLAIR and $\mathrm{dMRI}$ and functional acquisitions between the groups and with each other. For this purpose appropriate preprocessing and adequate analysis of data will also be performed.

\section{Discussion}

This protocol describes a RCT to test whether cognitive training $(30 \mathrm{~h} / 12$ weeks) exerts a positive effect not only on the cognitive and daily functioning of children with $\mathrm{CP}$ but also on other measures such as participation and QOL. We will further test whether there are changes in brain functions and structure following cognitive training.

This protocol has four key strengths. Firstly, it will be the first study of $\mathrm{CP}$ that analyses the functional and structural brain changes due to plasticity after performing cognitive training.

Secondly, it will be a single-blind RCT to avoid biases. Thirdly, the therapy will be home-based computerized multi-modal cognitive training, which provides the opportunity to increase the length of sessions and, at the same time, maximize training adherence [12]. Finally, this will be one of only a few studies so far published in CP to analyse the long-term results after training, providing information on the long-term neuropsychological effects and the maintenance of their benefits on daily functioning of children with $\mathrm{CP}$ but also on participation and QOL.

This protocol also has some limitations. First, the anticipated small sample size may be too small to detect brain changes. Consequently, to improve statistical power we will carefully consider the preparation of participants for scanning, and the image processing methods [31]. A further limitation is the lack of an active control group. This is due to the difficulty in finding or developing an alternative cognitive task completely free of EF training. Instead, this study will compare the effect of care as usual to the effect of adding a home-based computerized multi-modal cognitive training programme to determine what this additional therapy adds, if anything.

In conclusion, if this on-line and home-based training proves to be effective, it could be a cost-effective intervention for children with $\mathrm{CP}$ and their families, with near effects on EF and far effects on memory and visuospatial functions and on participation or QOL that are easily accessible for all.

\section{Supplementary information}

Supplementary information accompanies this paper at https://doi.org/10. 1186/s12887-019-1904-X.

Additional file 1. Motor, communication, intelligence and other measures at T0 assessments. Table with name and brief description of each assessment tool in T0. Neuropsychological tests and parental questionnaires at T0, T1 and T2 assessments. Table with name, brief description and reliability information of each assessment tool at T0, T1 and T2.

\section{Abbreviations}

CP: Cerebral Palsy; EF: Executive Function; GEEs: Generalized Estimating Equations.; IQ: Intelligence Quotient; MACS: Manual Abilities Classification System; MRI: Magnetic Resonance Imaging; QoL: Quality Of Life; RCT: Randomized Controlled Trial; UB: University of Barcelona

\section{Acknowledgements}

We would like to thank all the children and parents who currently participate in this study. We would also like to thank the European Academy of Childhood Disability for selecting our abstract to be presented in the 30th Annual Meeting [48].

\section{Authors' contributions}

The study protocol was designed by all the authors. RP will provide supervision throughout the study as a lead investigator. MG-G and MB are responsible for the ethics application and reporting. ET-T, M-MP, FG and XA will be responsible for patient selection. $M G-G$ and $M B$ will be responsible for recruitment and data collection. $\mathrm{DL}$ will take on a lead role in the data management. MG-G and MB will take a lead role in preparing publications. $\mathrm{RP}, \mathrm{OL}-\mathrm{H}$ and JB-P will contribute to the preparation of publications. XC, JM, $M C, E S, K P, L R$ and $R B$ will contribute with advice during the study in their specific fields. MG-G and MB will use data from this study to contribute to their PhD theses. MG-G drafted the final version of this manuscript, while all authors critically reviewed and approved the final version.

\section{Authors' information}

Not applicable

\section{Funding}

This project was supported by the Ministerio de Economía y Competitividad (PSI2016-75979-R AEI/FEDER, UE). The funding body peer reviewed the design of the study and collection, analysis and interpretation of data but not in writing manuscript as a part of the grant award project. The funding body also accepted any change made after this first peer review process. The following funding bodies did not participate in the design of the study and collection, analysis, and interpretation of data and in writing the manuscript.

This project was also supported by the Agència de Gestió d'Ajuts Universitaris i de Recerca from Generalitat de Catalunya (2017SGR0748). Montse Blasco received a research grant from Universitat de Barcelona (grant code APIF_2018-2019).

Lee Reid is funded through an Advance Queensland Research Fellowship (R-09964001).

\section{Availability of data and materials}

Not applicable, as this is a protocol manuscript.

\section{Ethics approval and consent to participate}

Signed consent will be obtained from all participants' parents or guardians by paper. Verbal consent will be obtained from children. The study has been approved by the University of Barcelona's Institutional Ethics Committee, Institutional Review Board (IRB 00003099, assurance number: FWA00004225; http://www.ub.edu/recerca/comissiobioetica.htm). The research will be conducted in accordance with the Helsinki Declaration. Findings will be disseminated through local seminars, international conferences and peerreviewed journals.

Manuscript is adhered to SPIRIT guidelines for protocols.

Trial registration: NCT0402574. 


\section{Consent for publication}

Not applicable.

\section{Competing interests}

The authors declare that they have no competing interests.

\section{Author details}

'Departament de Psicologia Clínica i Psicobiologia, Universitat de Barcelona, Passeig Vall d'Hebron 171, Barcelona 08035, Spain. 'nstitut de Neurociències, Universitat de Barcelona, Passeig Vall d'Hebron 171, Barcelona 08035, Spain. ${ }^{3}$ Institut de Recerca Sant Joan de Déu, Passeig de Sant Joan de Déu 2, Barcelona 08950, Spain. ${ }^{4}$ Australian e-Health Research Centre, Commonwealth Scientific and Industrial Research Organisation, Brockway 65, Brisbane 6014, Queensland, Australia. ${ }^{5}$ Queensland Cerebral Palsy and Rehabilitation Research Centre, The University of Queensland, Graham 62, Brisbane 4101, Queensland, Australia. ${ }^{6}$ Departament de Psicologia Social i Psicologia Quantitativa, Universitat de Barcelona, Barcelona 08035, Spain. ${ }^{7}$ Servei de Neurologia, Hospital Universitari Sant Joan de Déu, Passeig Sant Joan de Déu 2, Barcelona 08950, Spain. ${ }^{8}$ Servei de Rehabilitació i Medicina Física, Hospital Universitari Vall d'Hebron, Passeig Vall d'Hebron 119-129, Barcelona 08035, Spain. ${ }^{9}$ Serveis de Rehabilitació, Associació de la Paràlisi Cerebral (ASPACE), Camí Tres Pins 31-35, Barcelona 08038, Spain.

${ }^{10}$ Departament de Cognició, Desenvolupament i Psicologia de l'Educació, Universitat de Barcelona, Passeig Vall d'Hebron 171, Barcelona 08035, Spain.

${ }^{11}$ Unitat de Tècniques Augmentatives de Comunicació (UTAC), Universitat de Barcelona, Passeig Vall d'Hebron 171, Barcelona 08035, Spain.

Received: 13 November 2019 Accepted: 24 December 2019 Published online: 07 January 2020

\section{References}

1. Rosenbaum P, Paneth N, Leviton A, Goldstein M, Bax M, Damiano D, et al. A report: the definition and classification of cerebral palsy April 2006. Dev Med Child Neurol. 2007:49(s109):8-14

2. Oskoui M, Coutinho F, Dykeman J, Jetté N, Pringsheim T. An update on the prevalence of cerebral palsy: a systematic review and meta-analysis. Dev Med Child Neurol. 2013;55(6):509-19.

3. Gosling AS. Recent advances in the neuroimaging and neuropsychology of cerebral palsy. Appl Neuropsychol Child. 2017;6(1):55-63.

4. Colver A, Rapp M, Eisemann N, Ehlinger V, Thyen U, Dickinson HO, et al. Self-reported quality of life of adolescents with cerebral palsy: a crosssectional and longitudinal analysis. Lancet. 2015;385(9969):705-16.

5. Straub K, Obrzut JE. Effects of cerebral palsy on neuropsychological function. J Dev Phys Disabil. 2009;21:153.

6. Stadskleiv K, Jahnsen R, Andersen GL, von Tetzchner S. Neuropsychological profiles of children with cerebral palsy. Dev Neurorehabil. 2018;21(2):108-20.

7. Ego A, Lidzba K, Brovedani P, Belmonti V, Gonzalez-Monge S, Boudia B, et al. Visual- perceptual impairment in children with cerebral palsy: a systematic review. Dev Med Child Neurol. 2015;57(s2):46-51.

8. Pirila S, van der Meere JJ, Rantanen K, Jokiluoma M, Eriksson K. Executive functions in youth with spastic cerebral palsy. J Child Neurol. 26(7):817-21.

9. Bottcher L. Children with spastic cerebral palsy, their cognitive functioning, and social participation: a review. Child Neuropsychol. 2010;16(3):209-28

10. Laporta-Hoyos O, Ballester-Plané J, Póo P, Macaya A, Meléndez-Plumed M, Vázquez $\mathrm{E}$, et al. Proxy-reported quality of life in adolescents and adults with dyskinetic cerebral palsy is associated with executive functions and cortical thickness. Qual Life Res. 2017;26(5):1209-22.

11. Novak I, Mcintyre S, Morgan C, Campbell L, Dark L, Morton N, et al. A systematic review of interventions for children with cerebral palsy: state of the evidence. Dev Med Child Neurol. 2013;55(10):885-910.

12. Simons DJ, Boot WR, Charness N, Gathercole SE, Chabris CF, Hambrick DZ, et al. Do "brain-training" programs work? Psychol Sci Public Interest. 2016; 17(3):103-86.

13. Conklin HM, Ogg RJ, Ashford JM, Scoggins MA, Zou P, Clark KN, et al. Computerized cognitive training for amelioration of cognitive late effects among childhood cancer survivors: a randomized controlled trial. J Clin Oncol. 2015;33(33):3894-902.

14. Daley D, Van Der Oord S, Ferrin M, Danckaerts M, Doepfner M, Cortese S, et al. Behavioral interventions in attention-deficit/hyperactivity disorder: a meta-analysis of randomized controlled trials across multiple outcome domains. J Am Acad Child Adolesc Psychiatry. 2014;53(8):835-47.

15. Davis JC, Marra CA, Najafzadeh M, Liu-Ambrose T. The independent contribution of executive functions to health related quality of life in older women. BMC Geriatr. 2010;10:16.

16. Eve M, O'Keeffe F, Jhuty S, Ganesan V, Brown G, Murphy T. Computerized workingmemory training for children following arterial ischemic stroke: a pilot study with long-term follow-up. Appl Neuropsychol Child. 2016;5(4):273-82.

17. Hardy KK, Willard WW, Allen TM, Bonner MJ. Working memory training in survivors of pediatric cancer: a randomized pilot study. Psychooncology. 2013;22(8):1856-65.

18. McBride RL, Horsfield S, Sandler CX, Cassar J, Casson S, Cvejic E, et al. Cognitive remediation training improves performance in patients with chronic fatigue syndrome. Psychiatry Res. 2017;257:400-5.

19. Best MW, Gale D, Tran T, Haque MK, Bowie CR. Brief executive function training for individuals with severe mental illness: effects on EEG synchronization and executive functioning. Schizophr Res. 2019;203:32-40.

20. Damholdt MF, Mehlsen M, O'Toole MS, Andreasen RK, Pedersen AD, Zachariae R. Web-based cognitive training for breast cancer survivors with cognitive complaints-a randomized controlled trial. Psychooncology. 2016; 25(11):1293-300.

21. De Giglio L, De Luca F, Prosperini L, Borriello G, Bianchi V, Pantano P, et al. A low-cost cognitive rehabilitation with a commercial video game improves sustained attention and executive functions in multiple sclerosis: a pilot study. Neurorehabil Neural Repair. 2015;29(5):453-61.

22. Eggenberger $P$, Wolf $M$, Schumann $M$, de Bruin ED. Exergame and balance training modulate prefrontal brain activity during walking and enhance executive function in older adults. Front Aging Neurosci. 2016;8:66.

23. Diamond A. Executive Functions. Annu Rev Psychol. 2013;64:135-68.

24. Løhaugen GC, Beneventi H, Andersen GL, Sundberg C, Østgård HF, Bakkan E, et al. Do children with cerebral palsy benefit from computerized working memory training? Study protocol for a randomized controlled trial. Trials. 2014;15:269.

25. Mak C, Whittingham $\mathrm{K}$, Ross C, Boyd RN. Effect of mindfulness yoga programme MiYoga on attention, behaviour, and physical outcomes in cerebral palsy: a randomized controlled trial. Dev Med Child Neurol. 2018;60(9):922-32.

26. Cortese S, Ferrin M, Brandeis D, Buitelaar J, Daley D, Dittmann RW, et al. Cognitive training for attention-deficit/hyperactivity disorder: meta-analysis of clinical and neuropsychological outcomes from randomized controlled trials. J Am Acad Child Adolesc Psychiatry. 2015;54(3):164-74.

27. Hardy JL, Nelson RA, Thomason ME, Sternberg DA, Katovich K, Farzin F, et al. Enhancing Cognitive Abilities with Comprehensive Training: A Large, Online, Randomized, Active-Controlled Trial. PLoS One. 2015;10(9):e0134467.

28. Piovesana AM, Ross S, Whittingham K, Ware RS, Boyd RN. Stability of executive functioning measures in 8-17-year-old children with unilateral cerebral palsy. Clin Neuropsychol. 2015;29(1):133-49.

29. Boyd RN, Mitchell LE, James ST, Ziviani J, Sakzewski L, Smith A, et al. Move it to improve it (Mitii): study protocol of a randomised controlled trial of a novel web-based multimodal training program for children and adolescents with cerebral palsy. BMJ Open. 2013;3:e002853.

30. Bilde PE, Kliim-Due M, Rasmussen B, Petersen LZ, Petersen TH, Nielsen JB. Individualized, home-based interactive training of cerebral palsy children delivered through the internet. BMC Neurol. 2011;11:32.

31. Reid LB, Rose SE, Boyd RN. Rehabilitation and neuroplasticity in children with unilateral cerebral palsy. Nat Rev Neurol. 2015;11(7):390-400.

32. Manning KY, Fehlings D, Mesterman R, Gorter JW, Switzer L, Campbell C, et al. Resting state and diffusion neuroimaging predictors of clinical improvements following constraint-induced movement therapy in children with hemiplegic cerebral palsy. J Child Neurol. 2015;30(11):1507-14.

33. Inguaggiato E, Sgandurra G, Perazza S, Guzzetta A, Cioni G. Brain reorganization following intervention in children with congenital hemiplegia: a systematic review. Neural Plast. 2013;2013:356275.

34. Horowitz-Kraus T, Toro-Serey C, Difrancesco M. Increased resting-state functional connectivity in the cingulo-opercular cognitive-control network after intervention in children with reading difficulties. PLoS One. 2015;10(7): e0133762.

35. Astle DE, Barnes JJ, Baker K, Colclough GL, Woolrich MW. Cognitive training enhances intrinsic brain connectivity in childhood. J Neurosci. 2015;35(16): 6277-83.

36. Chen N, Cai P, Zhou T, Thompson B, Fang F. Perceptual learning modifies the functional specializations of visual cortical areas. Proc Natl Acad Sci U S A. 2016;113(20):5724-9. 
37. Suzuki T, Takeuchi S, Yoshinaga H. Dislocation dynamics and plasticity Springer series in materials science v. 12. Berlin: Springer; 1991.

38. Frantzidis C, Moraitou D, Ladas AK, Anderson-Hanley C, Barcelos NM, Zimmerman EA, et al. The Aerobic and Cognitive Exercise Study (ACES) for Community-Dwelling Older Adults With or At-Risk for Mild Cognitive Impairment (MCl): Neuropsychological, Neurobiological and Neuroimaging Outcomes of a Randomized Clinical Trial. 2019;1:76.

39. Eliasson A-C, Krumlinde-Sundholm L, Rösblad B, Beckung E, Arner M, Öhrvall A-M, et al. The manual ability classification system (MACS) for children with cerebral palsy: scale development and evidence of validity and reliability. Dev Med Child Neurol. 2006;48(7):549-54.

40. Toronto AS. Screening test of Spanish grammar. Illinois: Northwestern University Press; 1973.

41. Sabbadini M, Bonanni R, Carlesimo GA, Caltagirone C. Neuropsychological assessment of patients with severe neuromotor and verbal disabilities. J Intellect Disabil Res. 2001;45(2):169-79.

42. Ballester-Plané J, Laporta-Hoyos O, Macaya A, Póo P, Meléndez-Plumed M, Vázquez É, et al. Measuring intellectual ability in cerebral palsy: the comparison of three tests and their neuroimaging correlates. Res Dev Disabil. 2016;56:83-98.

43. Mclean B, Blakeman M, Carey L, Ward R, Novak I, Valentine J, et al. Discovering the sense of touch: protocol for a randomised controlled trial examining the efficacy of a somatosensory discrimination intervention for children with hemiplegic cerebral palsy. BMC Pediatr. 2018;18(1):252.

44. PREBO-6: Prediction of childhood Brain Outcomes in infants born preterm using neonatal MRI and concurrent clinical biomarkers [Internet]. ANZCTR. [cited 18 Sep 2019]. Available from: https://anzctr.org.au/Trial/Registration/ TrialReview.aspx?ACTRN $=12619000155190$

45. Boyd RN, Davies PS, Ziviani J, Trost S, Barber L, Ware R, et al. PREDICT-CP: study protocol of implementation of comprehensive surveillance to predict outcomes for school-aged children with cerebral palsy. BMJ Open. 2017; 7(7):e014950.

46. Sakzewski L, Bleyenheuft Y, Boyd RN, Novak I, Elliott C, Reedman S, et al. Protocol for a multisite randomised trial of hand-arm bimanual intensive training including lower extremity training for children with bilateral cerebral palsy: HABIT-ILE Australia. BMJ Open. 2019;9(9):e032194.

47. Reid LB, Pagnozzi AM, Fiori S, Boyd RN, Dowson N, Rose SE. Measuring neuroplasticity associated with cerebral palsy rehabilitation: an MRI based power analysis. Int J Dev Neurosci. 2017:58:17-25.

48. García-Galant M, Cañas A, Blasco M, Laporta-Hoyos O, Ballester-Plané J, Caldú X, Miralbell J, Leiva D, Toro-Tamargo E, Alonso X, Meléndez-Plumed M, Gimeno F, Pannek K, Boyd NR, Pueyo R. Study protocol of a randomised controlled trial of online executive function training for children with cerebral palsy. Dev Med Child Neurol. 2018;60(s2):38.

\section{Publisher's Note}

Springer Nature remains neutral with regard to jurisdictional claims in published maps and institutional affiliations.

\section{Ready to submit your research? Choose BMC and benefit from:}

- fast, convenient online submission

- thorough peer review by experienced researchers in your field

- rapid publication on acceptance

- support for research data, including large and complex data types

- gold Open Access which fosters wider collaboration and increased citations

- maximum visibility for your research: over $100 \mathrm{M}$ website views per year

At $\mathrm{BMC}$, research is always in progress.

Learn more biomedcentral.com/submissions 